\title{
Erratum
}

\section{An Efficient Preparation of 2-Imidazolines and Imidazoles from Aldehydes with Molecular Iodine and (Diacetoxyiodo)benzene}

Midori Ishihara, Hideo Togo* Synlett 2006, 227.

In Scheme 1, the stereochemistry of $(R, R)-(+)$-diphenylethylenediamine, and that of the resulting product, was incorrect. The corrected scheme is shown here:

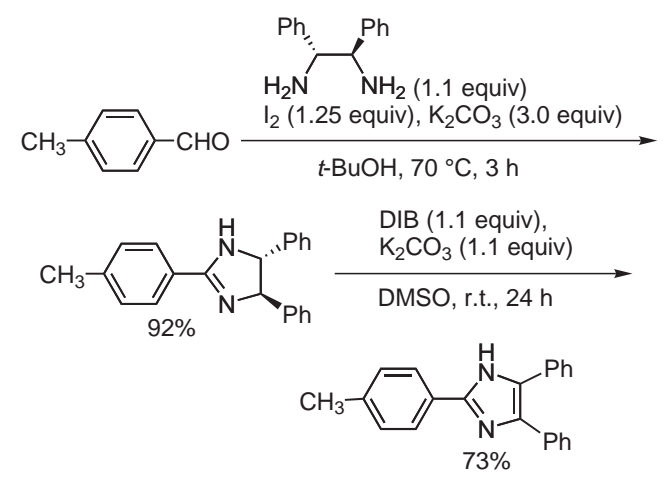

Scheme 1 